\title{
An Evaluation of Authoring Interfaces for Image-based Navigation
}

\author{
Benjamin \\ Walther-Franks \\ TZI, Universität Bremen \\ Bremen, Germany \\ bwf@tzi.de
}

\author{
Dirk Wenig \\ TZI, Universität Bremen \\ Bremen, Germany \\ dwenig@tzi.de
}

\author{
Rainer Malaka \\ TZI, Universität Bremen \\ Bremen, Germany \\ malaka@tzi.de
}

\author{
Barbara Grüter \\ Hochschule Bremen \\ Bremen, Germany \\ grueter@informatik.hs- \\ bremen.de
}

\begin{abstract}
We present the development and evaluation of an authoring system for image-based pedestrian navigation which lets authors take pictures and annotate instructions on the go in three interface variants. Results indicate that a freehand manner of photograph annotation is fastest, while authors strive toward visually pleasing annotation compositions.
\end{abstract}

\section{Categories and Subject Descriptors}

H.5.2 [Information Interfaces and Presentation]: User Interfaces-Evaluation/methodology, Graphical user interfaces (GUI), Screen design, Interaction styles

\section{General Terms}

Human Factors, Experimentation

\section{Keywords}

mobile navigation, evaluation, user-created content

\section{INTRODUCTION}

Advantages of an image-based approach to pedestrian navigation systems has been demonstrated in several studies $[1,2,3]$. Encouraging users to create image content for such systems is a possible solution to the lack of image databases. While there are efforts to open up the content map-based systems rely upon ${ }^{1}$, to our knowledge, the only existing product that supports users in creating image content for navigation is the application BreadCrumbz ${ }^{2}$, and no scientific investigation of user-created content for image-based navigation has been made so far. In the following we present the design and evaluation of three interface alternatives for annotating photographs on the go in order to create route instructions for pedestrian navigation: Separate annotation with icons, perspective overlay annotation and freehand overlay annotation.

\footnotetext{
${ }^{1}$ for example http://www.openstreetmap.org

${ }^{2}$ http: //www. bcrumbz.com/
}

Copyright is held by the author/owner(s).

MobileHCI'09, September 15 - 18, 2009, Bonn, Germany. ACM 978-1-60558-281-8.

\section{MOBILE ROUTE AUTHORING}

For our authoring system, users should be able to take photographs and annotate navigation information themselves. As the visualisation largely determines the annotation interface, we picked three approaches that all transport comparable information. Inspired by route planners and the BreadCrumbz application, separate annotation provides photographs alongside text and iconic direction instructions (Figure 1). Perspective annotation (Figure 2a and 2b) integrates annotations into the source image, in order to create visualisations analogous to simplified augmented reality [3] visualisations. Freehand annotation offers the most freedom for authors, allowing them to draw or write onto a photograph with a stylus pen in the manner of a simple painting application (Figure 2c).
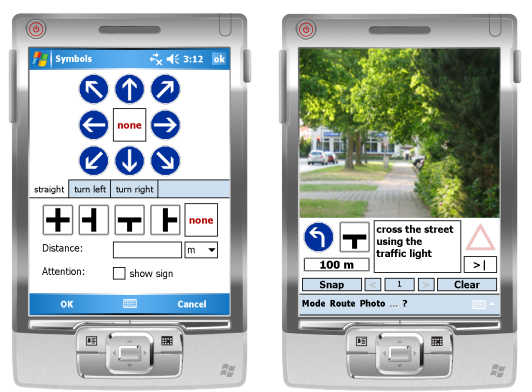

Figure 1: Separate annotation author and viewer modes.

For the first two annotation interfaces we had to restrict the set of instructions the author could annotate to a degree that users were able to handle but which would still let them express themselves. As we are not aware of any standards for which navigation elements to include we determined common functionality for the other variants by conducting a small-scale explorative study with the freehand variant before the other two were fully implemented. The study showed that the types of annotated visual route instructions used most often were arrows in various shapes, text, and highlights in the form of boxes or circles around objects. Additional lines were also used to clarify the meaning of an arrow relative to the path layout.

\section{EVALUATION}

We evaluated the authoring mode of our system in a field setting. The goal was to compare the three authoring in- 


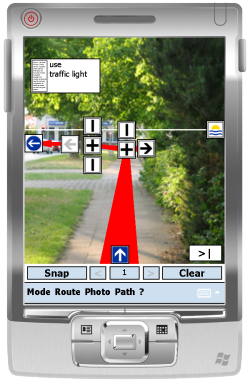

(a)

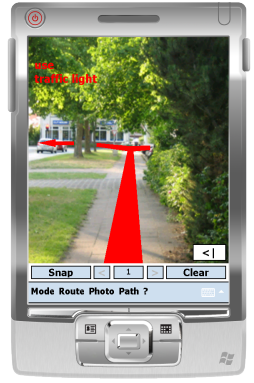

(b)

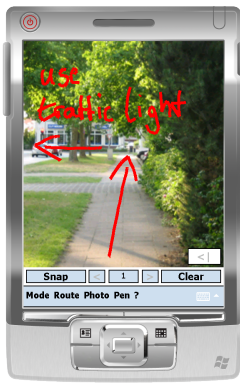

(c)
Figure 2: Perspective annotation author (2a) and viewer (2b) modes; Freehand annotation interface (2c).

terfaces regarding usability. We selected three groups of five participants with overall comparable age and experience with mobile navigation systems. We chose a route of about 750 metres length that included all the major route situations the system should cope with. The participants were asked to create, with the help of the authoring system, complete route instructions by taking photographs and annotating them such that a user unknown to the area and route could find their way from start to end by incrementally navigating from waypoint to waypoint.

The test runs took between 12 and 36 minutes. Participants using the freehand interface were significantly faster than those using the fixed layout or sAR interface. The corrections made on average by users of the perspective annotation were significantly higher than the amount of corrections made by users in other groups. These numbers must be taken with a grain of salt, as we were unable to normalise the classification of what is a correction and what is not. However, even a less strict definition or weighting would probably have shown similar results. The amount of photos taken varied a lot, with a minimum of 7 per route, a maximum of 18 , and an average of 10.8 , which corresponds roughly with the 12 decision points of the route. Although it was never mentioned as part of the task, participants automatically tried to achieve the "nicest" or "clearest" results possible. At the end of the test run participants were confronted with the two interfaces alternative to the one used. None favoured the separate annotation over the others and the perspective interface was mentioned as more favourable than other systems. Despite changeable winter weather all participants mentioned that they enjoyed creating the route.

Times and correction rates provide a clear picture: The freehand annotation is by far the fastest to use and significantly less corrections were needed per waypoint. Users of the separate annotation took about twice as long on average and the perspective annotation took users about five times as long. Clearly, with this interface variant, users created the most input, as is reflected in the times as well. This was not obvious, as it also allows creating single arrows with one tap of the screen. Apparently the perspective annotation invited users to fine-tune the route depictions, creating visually pleasing annotations. The results of a usability questionnaire did not demonstrate significant differences between
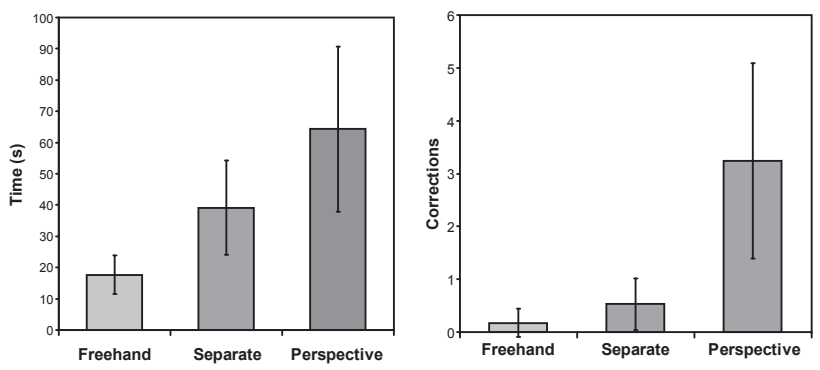

Figure 3: Image annotation mean times and corrections per group. Vertical lines indicate the standard deviation.

groups. Observation and feedback garnered in interviews suggest that users put much importance on the quality of their photographs and annotations. The fixed layout interface was apparently the least favourable and the perspective variant the most favourable, as users seemed to tend toward the aesthetically more pleasing visualisations. Thus user comments cast a different light on the significance of duration of the editing task: For an authoring scenario, time and precision possibly aren't always the determining factors, it is rather the expressiveness and clarity of the results produced.

\section{CONCLUSION}

The measured times of completion and correction rates for annotating photographs clearly show that the freehand annotation is superior. However, qualitative insights gained in observation and interviews imply that participants put a high importance on the visual clarity and perfection of their annotations and seemed to favour annotation in perspective despite a more complex editing process. The results favour a visual integration of image and instructions and freehand editing over constrained editing, providing an indication for designers and developers of mobile pedestrian navigation systems employing user-created content. In the future we plan to evaluate the visualisations produced by the three interfaces from a viewer perspective, in order to fully assess the trade-offs between ease of use for route authors and route followers in image-based pedestrian navigation.

\section{Study partly sponsored by the Klaus Tschira Foundation.}

\section{REFERENCES}

[1] L. Chittaro and S. Burigat. Augmenting audio messages with visual directions in mobile guides: an evaluation of three approaches. In M. Tscheligi, R. Bernhaupt, and K. Mihalic, editors, Mobile HCI, pages 107-114. ACM, 2005.

[2] T. H. Kolbe. Augmented videos and panoramas for pedestrian navigation. In G. Gartner, editor, Proceedings of the 2nd Symposium on Location Based Services and TeleCartography 2004 on 28.-29. January in Vienna. TU Wien, 2004.

[3] B. Walther-Franks and R. Malaka. Evaluation of an augmented photograph-based pedestrian navigation system. In A. Butz, B. D. Fisher, A. Krüger, P. Olivier, and M. Christie, editors, Smart Graphics, volume 5166 of Lecture Notes in Computer Science, pages 94-105. Springer, 2008. 